\title{
MEDICIÓN CIENTÍFICA Y EL CASO DE EINSTEIN CONTRA LORENTZ
}

Miguel Agustín Aguilar Sandoval Universidad Nacional Autónoma de México miguelllzim@hotmail.com https://orcid.org/0000-0002-1014-5516

RESUMEn: A inicios del siglo XX, Albert Einstein y Hendrik Lorentz produjeron explicaciones diferentes acerca de los mismos fenómenos. Sin embargo, rápidamente se produjo un consenso, en favor de Einstein, que ha sido difícil de comprender para historiadores y filósofos de la ciencia. La literatura reciente explica ese éxito señalando conflictos entre algunas ideas de Lorentz y la temprana física cuántica. Sin negar que esos factores pudieron contribuir en la aceptación de la relatividad especial, propongo una explicación complementaria en la que atribuyo el éxito de Einstein al uso de la estabilidad de la medición de la velocidad de la luz.

PALABRAS CLAVE: relatividad, robustez, velocidad, luz, epistemología

SUMmaRY: At the beginning of the 20th century, Albert Einstein and Hendrik Lorentz produced different explanations for the same phenomena. However, there was a consensus in favor of Einstein, which has been difficult for historians and philosophers of science to understand. Recent literature explains that success by pointing out conflicts between some ideas of Lorentz and the early quantum physics. Despite the fact that those events could contribute to the acceptance of special relativity, I propose a complementary explanation in which I attribute Einstein's success to his use of the stability of the speed of light measurement.

KEY WORDS: relativity, robustness, speed, light, epistemology

\section{Introducción}

En 1905, Albert Einstein (1879-1955) propuso la teoría de la relatividad especial, la cual es reconocida como uno de los logros científicos más notables del siglo XX. Sin embargo, no parece que inicialmente hubiera evidencia empírica que favoreciera a la relatividad especial sobre otras propuestas de la época para explicar los mismos fenómenos. En particular, la electrodinámica clásica de Hendrik Antoon Lorentz (1853-1928) utilizaba hipótesis diferentes, pero recuperaba las mismas consecuencias empíricas. No obstante, se formó un claro consenso entre los físicos alemanes (Max Planck, Arnold Sommerfeld, Wilhelm Wien, Max Born, Paul Ehrenfest y Max Laue) en favor de la relatividad especial para 1911. Las razones de este éxito temprano de 
la relatividad especial han resultado difíciles de identificar y han generado debates para investigadores tales como Grünbaum (1959), Holton (1969), Zahar (1973a y 1973b), Feyerabend (1974), Miller (1981), Nugayev (1983), Goldberg (1984), Pyenson (1985), Brush (1999), Janssen (1995, 2002, 2019), Brown (2005), Camp (2011), Acuña y Dieks (2014), Norton (2008), Bradley (2019), entre otros. Esto muestra que no son obvios los factores que favorecieron a la relatividad especial sobre la electrodinámica de Lorentz. "Lo que requiere explicación no es una supuesta ceguera de los lectores de Einstein, sino los factores que finalmente hicieron a la propuesta de Einstein más atractiva que otras propuestas válidas" (Darrigol 2000 p. 392). ${ }^{2}$

La explicación más convincente del éxito de la relatividad especial señala una inconsistencia entre la electrodinámica de Lorentz y la física cuántica. Es decir, "Lo que eventualmente superó a la teoría de Lorentz fue la teoría cuántica, no la teoría de la relatividad" (Janssen 2002 , p. 431). Sin embargo, parece extraño concluir que el éxito de la relatividad, que transformaba profundamente nuestra manera de entender al espacio y el tiempo, sólo se debiera a que evitaba el conflicto con una teoría cuántica aun en los primeros estadios de su desarrollo; sobre todo si consideramos la opinión de científicos y filósofos de la época que percibieron algo radicalmente nuevo en la relatividad especial. Por ejemplo, John Dewey consideraba que:

No es exagerado decir que cualesquiera que deban ser los desarrollos futuros en los descubrimientos acerca de la luz, o incluso si los detalles de la teoría de la relatividad de Einstein sean desacreditados algún día, una revolución genuina, y una que no retrocederá, ha sido efectuada en la teoría del origen, naturaleza y prueba de las ideas científicas. (Dewey 1929, p. 146)

Es decir, para Dewey, la novedad en el pensamiento de Einstein iba más allá de dar cuenta de los fenómenos. Por su parte, P.W. Bridgman (1882-1961) consideraba que la relatividad especial era incluso más notable que la relatividad general. ${ }^{3}$ Para Bridgman la

${ }^{1}$ Las conclusiones de los autores mencionados son sorprendentemente diversas. David Cassidy, al revisar los trabajos de Goldberg (1984), Miller (1981) y Pyenson (1985) sobre este caso, comenta: "Los autores de los libros discrepan acerca de casi todo..." (Cassidy 1986, p. 178).

${ }^{2}$ La traducción de esta y las demás citas son mías, con excepción de aquellas en las que se indique el traductor en la bibliografía.

${ }^{3}$ Según Bridgman "El dictamen que este articulo presentaría contra Einstein es, por lo tanto, el siguiente: [...] él ha llevado a la teoría de la relatividad general 
novedad de la relatividad especial está en cómo asigna significado a los conceptos científicos a través de operaciones de medición. Aunque las ideas de Bridgman, como teoría del significado, resultaron poco convincentes, realmente parece que había una novedad en la manera en la que la relatividad especial relacionaba conceptos (como los de espacio y tiempo) con la medición.

Por lo anterior, vale la pena considerar si es posible dar una imagen diferente de este caso mediante un análisis de cómo Lorentz y Einstein utilizan los logros en medición producidos hasta su época. Un motivo adicional para esto es el interés que se ha generado en las últimas décadas en el estudio filosófico de las prácticas de medición, como se puede apreciar en los trabajos de Berka (1983), Mari (2003), Chang (2004, 2012), van Fraassen (2008), Tal (2012) o Guillaumin (2016). Estos estudios analizan, entre otras cosas, la relación entre conceptos científicos y prácticas de medición desde una perspectiva histórica, con lo que proveen interesantes conclusiones filosóficas acerca del crecimiento del conocimiento científico.

En otras palabras, haga a un lado por ahora el sorprendente contraste entre la manera en que los newtonianos empleaban el "tiempo" o el "espacio" a finales del siglo XVII y la manera en que los einstenianos usaban estos términos a inicios del siglo XX. Vea, en su lugar, la manera en que Poincaré, Bucherer, Einstein, Kaufmann y Lorentz coordinaron su uso de estos términos en experimentos particulares en entornos de laboratorio particulares. (Galison 1997, p. 48)

Así, el objetivo de este artículo es indagar si el estudio filosófico de la medición científica permite resaltar algún aspecto distintivo de la relatividad especial que explique su éxito sobre la electrodinámica de Lorentz. La práctica de medición más relacionada con la relatividad es la medición de la velocidad de la luz por lo que será el hilo conductor del análisis. Lo que mostraré es que dicha medición alcanzó, a finales del siglo XIX, una robustez mucho mayor que la medición de grandes distancias o la del tiempo; y que es la manera en que Einstein utiliza esa robustez lo que explica que la relatividad especial, a diferencia de propuestas como la de Lorentz, sobreviviera a los profundos cambios por los que atravesaría la física de las primeras décadas del siglo XX.

precisamente ese punto de vista acrítico pre-einsteiniano que tan convincentemente nos ha mostrado, en su teoría especial, lo que oculta la posibilidad de desastre" (Bridgman 1970, p. 354). 
Para mostrar lo anterior, señalaré, en la sección 2, algunos problemas en la medición de grandes distancias y la estandarización del tiempo, en la época anterior a Einstein, así como la confiabilidad que había en la medición de la velocidad de la luz. En la sección 3 resaltaré la notable robustez de la estabilidad que las prácticas de medición pueden alcanzar, lo que ha sido reconocido por una variedad importante de filósofos de la ciencia. En las siguientes dos secciones, 4 y 5 , expongo las propuestas de Lorentz y Einstein respectivamente a la luz de lo anterior. En la sección 6 comento algunos intentos de explicar el éxito de Einstein por parte de historiadores y filósofos. Finalmente, en la sección 7 doy una explicación complementaria que atribuye ese éxito al uso que hace Einstein de la robustez de la velocidad de la luz.

\section{Mediciones de grandes distancias, coordinación de relojes y la velocidad de la luz}

Desde el siglo XVIII las prácticas de medición de distancias, tiempos y de la velocidad de la luz han estado interrelacionadas. Esta relación comenzó por la necesidad de medir grandes distancias terrestres. Para determinar la distancia entre un observador y una posición de referencia se debía medir la latitud (distancia norte-sur) y la longitud (distancia este-oeste) terrestres. La primera se puede encontrar con observaciones de las estrellas (la estrella polar, por ejemplo, se ve más cerca del horizonte cuanto más cerca se está del ecuador). La longitud terrestre se puede deducir a partir de la diferencia de horarios. Sin embargo, saber el tiempo exacto que transcurría entre, por ejemplo, el amanecer en una región de la Tierra y el mismo evento en otra, sólo es posible si se tienen relojes coordinados en ambos lugares, lo cual era extremadamente difícil porque los relojes se desajustaban al ser transportados. Es decir, medir grandes distancias terrestres requería coordinar relojes colocados en los extremos de las distancias a medir. Cuando Galileo Galilei descubrió los satélites de Júpiter sugirió usarlos para este fin; registrando la hora en que, por ejemplo, uno de los satélites se ocultaba detrás de Júpiter y, conociendo la hora en que eso debía ocurrir en un meridiano de referencia, un observador podía deducir la longitud terrestre en que se encontraba. Sin embargo, pronto se reconocieron irregularidades en los movimientos de estos satélites. Ole Römer (1644-1710) notó que los satélites parecían adelantarse en su periodo cuando la Tierra estaba cerca de Júpiter, por lo que las irregularidades podían explicarse asumiendo que a la luz le toma tiempo llegar desde dichos satélites hasta la Tierra. Esa explicación fue corroborada por James Bradley (1693- 
1722) quien señaló un aparente movimiento en las estrellas a lo largo del año, conocido como aberración estelar, que podía explicarse como consecuencia de la combinación del movimiento de la Tierra con el de la luz. Los valores que ambos calcularon para el tiempo que a la luz debía tomarle recorrer una distancia como la órbita de la Tierra coincidían lo suficiente como para que otros astrónomos aceptaran sus conclusiones.

Sin embargo, ni Römer ni Bradley midieron propiamente la velocidad de la luz. Lo que ellos concluyeron fue que la luz necesita tiempo para atravesar una región del tamaño de la órbita de la Tierra. La distancia y el tiempo es lo que se necesita para determinar una rapidez, pero el valor de la distancia de la órbita de la Tierra era muy incierto en el siglo XVIII. Por ello, en la época se consideró que la aportación de ambos astrónomos era haber mostrado que la velocidad de la luz era finita, pero no medirla, ya que hacía falta saber cuál era la distancia que la luz recorre en el tiempo calculado.

Una manera de encontrar el tamaño de la órbita terrestre era mediante observaciones de la paralaje de Marte en oposición, pero éste resultó ser un método impreciso. El astrónomo Edmund Halley (1656-1742) y Joseph Nicholas Delisle (1688-1768) propusieron métodos diferentes basados en registrar los tránsitos de Venus (cuando Venus pasa entre la Tierra y el Sol) desde diferentes posiciones en la Tierra. Con dicha observación encontrar la distancia entre la Tierra y el Sol se convierte en un problema geométrico simple. Con todo, estos tránsitos no son comunes, por lo que no fue posible encontrar una buena estimación del tamaño de la órbita terrestre hasta los tránsitos de 1761 y 1769, y aun entonces los resultados fueron insatisfactorios. ${ }^{4}$

\subsection{La medición terrestre de la velocidad de la luz}

Fue hasta el siglo XIX que se produjo una medición de la rapidez de la luz con un método independiente del tamaño de la órbita de la Tierra. En 1849, Armand Hippolyte-Louis Fizeau (1819-1896) lo consiguió utilizando un dispositivo que consistía en una rueda dentada que se hacía girar a gran velocidad y un rayo de luz que se enviaba al espacio entre los dientes. Detrás de la rueda se colocaba un espejo, de manera que, la luz pasaba por el espacio entre los dientes de la rueda, se reflejase en el espejo y, si la rueda se había movido lo suficiente para eclipsar el rayo, entonces la velocidad de la luz podía

\footnotetext{
${ }^{4}$ Un estudio detallado de los problemas que enfrentaron los astrónomos del siglo XVIII para calcular un valor aproximado para la rapidez de la luz es Boyer 1941.
} 
determinarse en función de la velocidad angular de la rueda. Poco después, en 1862, Jean Bernard Léon Foucault (1819-1868) realizó su propia medición dirigiendo un rayo de luz hacía un espejo rotando a gran velocidad y midiendo la diferencia entre los ángulos con los que se reflejaba el rayo cuando el espejo estaba quieto y cuando giraba. Posteriormente, Alfred Cornu (1841-1902) obtuvo la rapidez de la luz con mayor precisión al mejorar el dispositivo de Fizeau, mientras que Albert A. Michelson (1852-1931) consiguió un resultado aún mejor con su modificación del dispositivo de Foucault.

\subsection{Mediciones terrestres contra mediciones astronómicas}

Mediciones como las de Fizeau y Foucault eran importantes debido a que había dudas sobre la precisión de los métodos astronómicos para medir la órbita de la Tierra. La idea era comparar la medición de la órbita de la Tierra obtenida con las observaciones de los tránsitos de Venus con la que se obtenía usando la medición terrestre de la velocidad de la luz y el tiempo que a ésta le toma atravesar dicha órbita. "Los astrónomos encontraron que cuando ellos usaban los valores para la rapidez de la luz, como habían sido calculados en los experimentos sobre la superficie de la Tierra, en sus fórmulas, los resultados no acordaban con cálculos u observaciones previas" (Canales 2015, p. 107). La imprecisión en los métodos astronómicos podía deberse a las discrepancias provocadas por el tiempo de reacción de los astrónomos, es decir, el tiempo que pasaba entre la observación y el registro de ésta. Se hicieron diferentes intentos por resolver esto. "Aun así, cronometrar el momento preciso de contacto entre dos cuerpos celestes probó ser tan difícil que algunos científicos, incluyendo al psicofísico belga Joseph Delboeuf, se vieron obligados a creer en una discontinuidad esencial en las percepciones" (Canales 2009, pp. 114-115). Otra posible fuente de error era el éter, que supuestamente llenaba el espacio. Para entonces, los estudios de Thomas Young (1773-1829) y, sobre todo, Augustin Fresnel (17881827) habían mostrado que la luz presenta propiedades de onda. De manera que se asumía que la luz era una onda que se producía en un medio llamado éter. Sin embargo, aún se debatía cómo afectaba el movimiento de la Tierra al éter y éste, a su vez, a las mediciones de la velocidad de la luz. Las hipótesis más importantes eran la del propio Fresnel y la de George Gabriel Stokes (1819-1903). De acuerdo con Fresnel, cuando la materia se mueve arrastra ligeramente al éter, es decir, le transmite una parte muy pequeña de su movimiento. Por otro lado, Stokes suponía que la materia arrastra por completo 
al éter, de la misma manera que la Tierra arrastra los gases de la atmósfera. Ambas hipótesis fueron pensadas para que recuperaran los resultados de Römer y Bradley, por lo que Fizeau buscó una manera de someterlas a prueba en un experimento terrestre. Dado que el éter es inobservable, Fizeau ideó un experimento para determinar si el agua en movimiento arrastra al éter, lo que haría que la velocidad de la luz cambiara en la misma proporción. En 1851, Fizeau encontró que la velocidad de la luz sí cambiaba, pero sólo por una fracción muy pequeña, misma que coincidía con el valor deducido por Fresnel.

Poco después James Clerk Maxwell (1831-1879) concluyó que la luz era una onda electromagnética, lo que fue corroborado por Heinrich Hertz (1857-1894). Esta conclusión mostraba que el mismo éter que explica la propagación de la electricidad y el magnetismo en el vacío era el que transmite a la luz, pero no explicaba cómo sería la interacción entre la materia y el éter. Maxwell consideraba que el éter debía ser como lo suponía Stokes y esperaba que experimentos más precisos que los de Fizeau le darían la razón.

\subsection{Los experimentos de Michelson sobre la velocidad de la luz}

Para investigar sobre el éter, Michelson diseñó un aparato llamado interferómetro. "El interferómetro era una maravilla. Inventado por el Michelson de veintiocho años en respuesta a un desafío de Maxwell, éste era capaz de revelar un efecto del orden de una parte en diez mil millones" (Holton 1969, p. 135). Este dispositivo separaba un rayo de luz en dos, mediante un espejo semitransparente, y luego los dirigía por caminos separados. Para determinar si había efectos del movimiento del éter respecto de la Tierra, uno de los rayos era dirigido en dirección paralela al movimiento de rotación de la Tierra y el otro en dirección perpendicular. Los rayos se reflejaban para hacerlos converger y, aprovechando el fenómeno conocido como interferencia de onda, Michelson podía determinar si la velocidad de alguno de los dos rayos había cambiado. Si el rayo dirigido en dirección del movimiento de la Tierra cambiaba su velocidad respecto al otro, se podía calcular el desfase observando movimiento en las franjas de interferencia. Michelson realizó su experimento en 1881. Como mencioné, según Fresnel, el éter es casi estacionario por lo que, al moverse la Tierra, éste tendría una velocidad relativa, lo que debía causar un desplazamiento notable de las franjas de interferencia. Sin embargo, no hubo el desplazamiento esperado. La conclusión fue que la hipótesis de Fresnel debía ser abandonada en favor de la de Stokes. Con todo, la hipótesis de Stokes seguía siendo difícil 
de reconciliar con el experimento de Fizeau en el que se medía la variación de la velocidad de la luz cuando ésta pasa a través de agua en movimiento. Por ello, Michelson repitió el experimento de Fizeau con ayuda de Edward Morley en 1886. Pero los resultados confirmaron los de Fizeau. En otras palabras, el experimento de 1881 llevó a Michelson a concluir: "La interpretación de estos resultados es que no hay desplazamiento de las bandas de interferencia. El resultado de la hipótesis de un éter estacionario se muestra, por tanto, incorrecta y la conclusión necesaria es que la hipótesis es errónea" (Michelson 1881 p. 128). Y del experimento de 1886 concluía: "El resultado de este trabajo es, por tanto, que el resultado anunciado por Fizeau es esencialmente correcto; y que el éter lumínico es enteramente inafectado por el movimiento de la materia que permea" (Michelson 1886, p. 386). La situación era más paradójica que nunca, el resultado de 1881 parecía demostrar que el éter se mueve junto con la Tierra y el de 1886 parecía implicar que eso no sucedía. Científicos como Lorentz y Alfred Potier (1840-1905) señalaron posibles fuentes de error en el experimento y pidieron una repetición. Con la ayuda de Morley y un interferómetro mejorado, Michelson repitió el experimento en 1887, pero sólo confirmó sus resultados previos.

\subsection{Prácticas de medición a finales del siglo XIX}

Mientras tanto, los astrónomos seguían encontrando inconsistencias entre sus cálculos y los resultados de experimentos terrestres.

Cálculos que involucraran longitudes eran un problema y también los que involucraban al tiempo. La falta de consenso en torno a los estándares astronómicos básicos sólo empeoró después de la investigación de Michelson [...] Para finales del siglo XIX, el desacuerdo en el valor exacto de la distancia Tierra-Sol permanecía enorme: cerca de 1,500,000 millas. (Canales 2015, p. 107)

Por otra parte, aunque la coordinación de relojes distantes se logró mediante el uso de señales eléctricas, definir la unidad estándar para medir el tiempo seguía siendo problemático. Uno puede determinar cuánto debe durar un segundo al dividir el día en veinticuatro horas, la hora en sesenta minutos y los minutos en sesenta segundos. Pero ¿cuánto dura el día que se debe dividir? El día solar cambia de duración a lo largo del año. Es por ello que en el siglo XIX se usaba el día sideral (a partir de las estrellas), pero para entonces había 
evidencia de que la Tierra desacelera en su rotación lo que afecta la duración del día sideral. ${ }^{5}$

En contraste, la situación de la medición de la velocidad de la luz era opuesta. Aunque Michelson y Morley no encontraron el movimiento de la tierra respecto del éter, sí contribuyeron a mostrar la estabilidad de la velocidad de la luz. "Mientras que las redes de comunicación electromagnética atravesaban cada vez más del mundo (tanto telegráficas como inalámbricas), los científicos se volvían cada más seguros de una cosa: el comportamiento de la luz en la superficie de la Tierra" (Canales 2015, p. 109). Los científicos se dieron cuenta de que los distintos métodos para medir la velocidad de la luz podían converger si se corregían los datos astronómicos con los experimentos terrestres. ${ }^{6}$ Esto tuvo como consecuencia una importante estabilidad en la medición de la velocidad de la luz. "La coordinación dinámica de conceptos cuantitativos con redes cada vez más robustas de instrumentos permite a los resultados de medición mantener su validez incluso cuando los estándares son mejorados o remplazados" (Tal 2012, p. 46). La confianza en la estabilidad de la velocidad de la luz llevó a Maxwell a proponer que los estándares para medición del espacio y el tiempo se basaran en ella. Incluso el filósofo Charles Sanders Peirce (1839-1914) menciona la medición de la velocidad de la luz para explicar su noción de realidad:

Un hombre puede investigar la velocidad de la luz al estudiar los tránsitos de Venus y la aberración estelar de las estrellas; otro por las oposiciones de Marte y los eclipses de los satélites de Júpiter; un tercero mediante el método de Fizeau; un cuarto por el de Foucault [...] Al principio puede que obtengan resultados distintos, pero conforme cada uno vaya perfeccionando su método y sus procedimientos, los resultados convergerán constantemente hacia un centro destinado [... ] La opinión destinada a ser aquella con la que todos los que investigan estarán de acuerdo finalmente es lo que entendemos por verdad, y el objeto representado en esta opinión es lo real. Así explicaría yo la realidad. (Peirce 1878, pp. 299-300)

En otras palabras, la tecnología permitió medir la velocidad de la luz con notable precisión y confianza, incluso aunque a nivel teórico hubiera muchas dudas acerca de la naturaleza de la luz y el éter.

${ }^{5}$ La preocupación de los investigadores de la época por este tema se puede notar, por ejemplo, en el ensayo de Poincaré (1913 [1898]).

${ }^{6}$ Le Verrier (1872) logró hacer coincidir los valores para la paralaje solar a partir de observaciones de distintos planetas. 
La situación era opuesta en los casos del espacio y del tiempo; no se cuestionaba la manera de concebirlos, pero sí los procesos para medir parámetros astronómicos, como el tamaño de la órbita de la Tierra, o la adecuación del reloj sideral como estándar para la medición del tiempo. Había acuerdo sobre como concebir el espacio y el tiempo, pero no en los procesos para su medición confiable.

\section{La robustez de la medición}

La estabilidad que alcanzó la medición de la velocidad de la luz era notablemente robusta y considero que reconocerlo puede cambiar la manera en que vemos las aportaciones posteriores de Lorentz y Einstein. Elaboraré esta afirmación en la sección 7, pero por el momento quiero señalar que esa robustez en las prácticas de medición es relativamente independiente de las teorías, lo cual ha sido reconocido por varios filósofos de la ciencia. Thomas Kuhn, por ejemplo, aunque afirmó que en los cambios de teoría podía haber pérdidas de poder explicativo, admitía "No sé de ningún caso en el desarrollo de la ciencia que muestre una pérdida de precisión cuantitativa a consecuencia de la transición de una teoría anterior a otra nueva" (Kuhn 1982, p. 236). También Herbert Feigl (1974) señaló que, si bien nuestras percepciones pueden tener carga teórica, las regularidades que encontramos entre ellas proporcionan una base relativamente estable para el desarrollo de la ciencia. En su investigación sobre la termometría Hasok Chang (2004) comenta que los fenómenos térmicos que se usaron para establecer los puntos fijos de las escalas de los termómetros del siglo XIX se siguieron usando a pesar de los profundos cambios en la comprensión teórica de la temperatura que se presentaron durante ese siglo.

La robustez de los puntos fijos proporcionó estabilidad a las observaciones cuantitativas, incluso cuando los cambios teóricos efectuaron cambios fundamentales en el mismo significado de esas observaciones. Los mismos números podían permanecer, lo que sea que "realmente significaran" $[\ldots]$

Aunque hoy es ampliamente aceptado que las observaciones están, de hecho, afectadas por las teorías que mantenemos; gracias a los conocidos y persuasivos argumentos elaborados en este sentido por Thomas Kuhn, Paul Feyerabend, Mary Hesse, Norwood Russell Hanson e incluso Karl Popper, así como por varios psicólogos empiristas; debemos tomar en serio el punto de Feigl de que no todas las observaciones son afectadas de la misma manera por cambios de paradigma u otras clases de cambios teóricos mayores. No importa que tan drásticamente cambien las teorías 
de alto nivel, algunas regularidades de nivel medio pueden permanecer relativamente inafectadas, incluso cuando su profundo significado teórico e interpretación cambie significativamente. (Chang 2004 pp. 51-52)

La robustez que las prácticas de medición pueden alcanzar no es dada, pero una vez que se alcanza ésta exhibe una peculiar estabilidad que proporciona una base empírica fija como la que se necesita para regular nuestro uso de conceptos teóricos o estabilizar otras prácticas. En el caso de la medición de la velocidad de la luz, ésta llegó a tener una estabilidad bastante robusta hacia finales del siglo XIX, por lo que se pensó que podía utilizarse para resolver las dificultades con las otras dos prácticas; la de medición de grandes distancias y la estandarización del tiempo. De hecho, Galison (2005) explica cómo el uso de señales electromagnéticas para coordinar relojes entre continentes mejoraba la medición de longitudes terrestres. Además, el tamaño de la órbita de la Tierra podía determinarse tomando la velocidad de la luz de los experimentos terrestres y el tiempo que los astrónomos calculaban que le toma recorrer una distancia como la de dicha órbita. También, Michelson utilizó interferómetros para determinar, con una precisión sin precedentes, qué tanto cambiaban de longitud las copias que se hacían del metro patrón para su distribución. La importancia de este trabajo era reconocida en la época, al punto de que Michelson recibió el premio nobel por sus aportaciones a la metrología, más que por su investigación respecto del éter. ${ }^{7}$ En cuanto a la estandarización del tiempo, J.C. Maxwell había señalado que la frecuencia de la misma podía usarse como estándar.

Sin embargo, para que la velocidad de la luz pudiera utilizarse con estos fines, había dificultades a nivel teórico y conceptual. Una primera dificultad era el éter; la velocidad de la luz sólo puede utilizarse para determinar el tamaño de la órbita terrestre si uno asume que el éter no afecta las mediciones. Pero una dificultad más fundamental tiene que ver con cómo entendemos el espacio y el tiempo, así como la manera en que la medición se relaciona con esa concepción. Medir implica coordinar un concepto cuantitativo (como el de longitud) con un proceso que consiste en un conjunto de operaciones físicas concretas (como hacer comparaciones de la longitud a determinar con una barra que se toma como estándar). Determinar si el proceso es adecuado depende, en parte, de nuestra concepción de aquello que medimos. Si consideramos que el espacio

${ }^{7}$ Véase Staley (2002) sobre la investigación de Michelson en metrología. 
no es el tipo de cosa que pueda variar con la temperatura buscaremos procesos de medición que ella no afecte. En el siglo XIX, y antes, se tenía una concepción del espacio y el tiempo que era independiente de quien la midiera y una concepción de la velocidad que sí dependía del movimiento de quien la medía. Así que se necesitaba determinar qué era lo que había que cambiar en nuestra concepción para que la velocidad de la luz pudiera usarse en las mediciones de grandes distancias y estandarizar el tiempo.

\section{La electrodinámica de H.A. Lorentz}

A finales del siglo XIX quedaban varios problemas teóricos respecto de la luz y la naturaleza del éter. En esta sección me ocuparé de la propuesta de solución de Hendrik Lorentz (1853-1928) y, en la sección siguiente, de la de Albert Einstein (1879-1955). Sin embargo, antes de continuar, quiero señalar que la comparación entre ambas propuestas puede complicarse innecesariamente si se hace en términos de teorías. Además, como el objetivo es identificar si las prácticas de medición pueden ofrecer elementos que permitan entender el eventual éxito de las ideas de Einstein, considero más adecuado utilizar categorías de análisis pensadas para el estudio de la medición científica. Un trabajo que propone categorías de análisis para estudiar la medición es el de Guillaumin (2016), que propone analizarla en términos de principios físicos, datos observacionales y modelos matemáticos. Siguiendo a Guillaumin, por modelos matemáticos me referiré a descripciones matemáticas. ${ }^{8}$ Por datos me referiré a " $[\ldots]$ observaciones, registros documentales de observaciones, mediciones directas realizadas con instrumentos, registros de tales observaciones etc." (Guillaumin 2016, p. 67). Finalmente, los principios físicos consisten en enunciados que "[...] se refieren de manera altamente abstracta, idealizada y/o general a objetos físicos, sus propiedades y/o sus relaciones con otros objetos" (Guillaumin 2016, p. 64; las cursivas son del original). Ya hemos visto los elementos que constituyen datos; los resultados de las observaciones y experimentos de Römer, Bradley, Fresnel, Fizeau, Foucault, Cornu, Weber, Maxwell y Michelson y Morley. Además, hemos visto principios físicos; i) la existencia del éter, ii) las propiedades que le atribuye Fresnel al éter, iii) las propiedades que le atribuye Stokes al éter, y iv) la identificación de la luz con las ondas electromagnéticas. De éstos, iv) estaba bien establecido y difícilmente había quien dudara de i).

${ }^{8}$ Cfr. Guillaumin 2016, pp. 69-73. 
Regresando a Lorentz, él propuso sus propios principios físicos y modelos matemáticos para dar cuenta de mediciones de diversos fenómenos electromagnéticos. En 1886, Lorentz proponía, como principio físico, entender al éter como un medio completamente estacionario, que no fuera arrastrado por el movimiento de cuerpos masivos como la Tierra. También asumía que todas las moléculas de cuerpos materiales poseen partículas microscópicas con carga eléctrica (lo que hoy llamamos electrones). Cuando las ondas de luz pasan a través de un material, como el agua, las vibraciones en el éter harían vibrar a las partículas cargadas de las moléculas de agua. Esto sucedería debido a que el éter, de acuerdo con Lorentz, no interactuaba con la materia, pero sí con efectos electromagnéticos. Las vibraciones de las partículas cargadas crearían nuevas ondas que interferirían con las ondas de la luz que pasaran a través de dicho medio. Esta interferencia explicaría que, al pasar por un medio más denso que el aire, la luz cambiara su velocidad de la manera que predice Fresnel y que había sido corroborada tanto por Fizeau, en 1851, como por Michelson y Morley en 1886.

Después, en 1892, Lorentz propuso un modelo matemático que consistía en una serie de transformaciones de coordenadas para describir fenómenos electromagnéticos, como la luz, en diferentes sistemas inerciales. En la física anterior se podía pasar una descripción elaborada desde un marco de referencia a otro utilizando transformaciones galileanas. Sin embargo, a finales del siglo XIX era claro que las ecuaciones de Maxwell para el campo electromagnético no son invariantes bajo transformaciones galileanas. Lorentz señaló que, dado un fenómeno electromagnético descrito en un sistema de referencia, se podía proponer otro marco de referencia ficticio que estuviera en reposo con respecto al éter. La descripción del fenómeno se pasaba al sistema de referencia ficticio mediante transformaciones distintas a las galileanas (transformaciones de Lorentz), se obtenía la solución al problema que se planteara y, finalmente, se obtiene la solución en el sistema real mediante transformaciones inversas. Se conoció como teorema de estados correspondientes al resultado con el que Lorentz mostraba que, a partir de la solución a un problema electrodinámico en su sistema ficticio, podía obtenerse una solución para el sistema real. Estas transformaciones de Lorentz incorporaban un parámetro llamado tiempo local que él entendía como una herramienta matemática necesaria para mantener invariantes las leyes de Maxwell, pero sin significado físico.

Con todo, para explicar experimentos más precisos que los de Fizeau, como el de Michelson y Morley, Lorentz necesitó una hipó- 
tesis adicional conocida como hipótesis de contracción; ésta asumía que las fuerzas intermoleculares se transmiten en el éter. Como consecuencia, cuando la Tierra se mueve a través del éter, los objetos se contraen en la dirección de dicho movimiento porque las fuerzas intermoleculares para objetos en movimiento se transforman del mismo modo que las fuerzas electromagnéticas. Así, Lorentz explicaba que Michelson no detectara cambios en la velocidad de la luz, ya que el brazo de su interferómetro se habría contraído dando la impresión de que la velocidad de la luz en esa dirección no cambiaba.

Lorentz presentó estas conclusiones de manera más sistemática en 1895 y, poco después, Henri Poincaré (1854-1912) señaló algunas dificultades. Poincaré consideraba insatisfactorio, por ejemplo, que la teoría de Lorentz violaba la tercera ley de Newton. Una solución del mismo Poincaré utilizaba el concepto de un flujo de éter, pero Lorentz no estaba convencido, ya que consideraba que el éter no era alterado. Eventualmente, Max Abraham resolvería este problema en 1902 usando el concepto de momento electromagnético. Pero desde 1899, Lorentz introdujo las versiones definitivas de las transformaciones coordinadas, el teorema de estados correspondientes y una versión más acabada de la hipótesis de contracción. Estos resultados llevarían a Lorentz a proponer un modelo para el electrón en 1904, el cual también Poincaré retomó en 1906.9

Gracias a estas aportaciones por primera vez había retroalimentación positiva entre datos, modelos y principios físicos en la medición de la velocidad de la luz. Es decir, los datos se podían predecir adecuadamente con los modelos y éstos, a su vez, mostraban las propiedades que los principios físicos afirmaban. Con todo, es importante notar que Lorentz mantenía el uso de los conceptos clásicos de espacio y tiempo, aun cuando los procesos para medir ambos habian mostrado menos confiabilidad que los métodos para medir la velocidad de la luz.

\section{La relatividad especial}

En 1905, Albert Einstein publicó un artículo titulado Sobre la electrodinámica de cuerpos en movimiento. En él, Einstein prescinde de principios físicos acerca del éter y, en su lugar, propone otros dos; el principio de relatividad y el principio de luz. El primero asumía que las leyes físicas no hacen distinción entre marcos de referencia inerciales. Es decir, todos los fenómenos físicos se pueden

\footnotetext{
${ }^{9}$ Una descripción más detallada de la teoría de Lorentz se encuentra en Michel Janssen 1995 y, más breve, en Acuña 2014.
} 
describir en términos de movimientos relativos. Este principio era resultado de las dificultades que se habían presentado para detectar el movimiento de la Tierra respecto al éter, las cuales "llevan a la conjetura de que ni los fenómenos de la mecánica, ni tampoco los de la electrodinámica tienen propiedades que correspondan al concepto de reposo absoluto" (Einstein 2005 [1905], pp. 399-400). El principio de luz afirmaba que la velocidad de la luz es independientemente de la velocidad de la fuente de emisión. En conjunto, estos principios generaban inconsistencias puesto que implicaban que la velocidad de la luz era la misma medida desde diferentes marcos de referencia. "Esta constancia de la velocidad de la luz, sin embargo, es incompatible con la ley de adición de velocidades conocida de la mecánica. ¿Por qué estas dos cosas se contradecían una a la otra? Sentí que había encontrado una dificultad extraordinaria aquí" (Einstein 2012 [1923], p. 637).

La inconsistencia que parecía generar el uso de estos principios era síntoma de un problema más profundo. " $[\ldots]$ inconsistencia interna en el conjunto de principios físicos indica un error grave en los criterios de evidencia que se utilizan para apoyar a los principios físicos" (Guillaumin 2016, p. 82; las cursivas son del original). La solución de Einstein emergió al analizar el concepto de tiempo.

Pasé casi un año en vano tratando de modificar la idea de Lorentz con la esperanza de resolver este problema [...] Mi solución, de hecho, tuvo que ver con el concepto de tiempo. El punto es que el tiempo no puede ser definido de manera absoluta, sino que hay una conexión inseparable entre el tiempo y la velocidad de una señal. Usando esta idea, pude, ahora por primera vez, resolver completamente la dificultad extraordinaria que había tenido antes. (Einstein 2012 [1923], p. 637)

\section{De acuerdo con Einstein}

Debemos tener en cuenta que todos nuestros juicios que implican al tiempo son siempre juicios sobre sucesos simultáneos. Si, por ejemplo, yo digo que "El tren llega aquí a las 7 en punto", eso significa, más o menos, "La manecilla pequeña de mi reloj apuntando a las 7 y la llegada del tren son sucesos simultáneos". (Einstein 2005 [1905], p. 401; las cursivas son del original.)

Este análisis le permite concluir que sólo podemos establecer la simultaneidad de eventos alejados mediante relojes coordinados. La coordinación de relojes puede llevarse a cabo mediante señales lumínicas, ya que su velocidad se supone constante. La medición del 
espacio y del tiempo queda definida de manera que siempre se mida el mismo valor para la velocidad de la luz. En consecuencia, aunque dos observadores tengan sus relojes sincronizados en un marco de referencia, no necesariamente estarán sincronizados en otro. Por lo tanto, no podemos asignar un significado absoluto al concepto de simultaneidad. El tiempo y la distancia pueden dilatarse o contraerse dependiendo de la velocidad del observador que realiza la medición. Desde una perspectiva epistemológica, la novedad consiste en señalar que algo que previamente se veía como justificado en realidad no lo estaba; el carácter absoluto del espacio y del tiempo. Es decir, la inconsistencia entre el principio de relatividad y el principio de luz lleva a Einstein a revisar los criterios de evidencia en los que se apoya la concepción del carácter absoluto del espacio y el tiempo. Al no encontrar un apoyo adecuado para dicha concepción, él introduce un requerimiento de acuerdo con el cuál la definición de los conceptos debe estar regulada por operaciones de medición. De manera que conceptos como el de espacio y tiempo pasan a ser conceptos métricos en la relatividad especial, es decir, quedan redefinidos a partir de operaciones de medición. ${ }^{10}$ Por lo anterior, considero que lo que hace Einstein representa una instancia de lo que Guillaumin (2016) llama crisis epistémica, la cual consiste en la modificación de los elementos de justificación de los principios físicos.

Al cambiar los principios físicos junto con sus elementos de justificación, Einstein pudo deducir un modelo matemático que incluye a las transformaciones de Lorentz (de 1899 y 1904), lo que le permite rescatar los resultados de Lorentz en cada experimento sobre electrodinámica, pero sin recurrir al éter. Además, el trabajo de Einstein también ofreció una solución a las dificultades de la época en torno a las prácticas de medición de tiempo y distancia.

La solución de Einstein a los problemas científicos de su era involucraron considerar que tanto las unidades de tiempo como de distancias se dilatan bajo ciertas circunstancias [...] Científicos y filósofos eran conscientes de que podían adoptar un sistema diferente para definir las unidades de tiempo y distancia de maneras que compensaran estos efectos de dilatación. O podían aceptar la brillante oferta de Einstein. La mayoría de ellos lo hicieron. (Canales 2015, p. 112)

\footnotetext{
${ }^{10}$ Con todo, hay que reconocer que "[...] incluso las definiciones firmes sólo regulan significados; no los agotan" (Chang 2004, p. 150).
} 


\section{Explicaciones del éxito de Einstein sobre Lorentz}

Tanto Lorentz como Einstein utilizaban las mismas ecuaciones, sin embargo, sus posturas tenían importantes diferencias. Por ejemplo, el éter es innecesario para Einstein, pero no para Lorentz. Más importante aún, Einstein entendía los conceptos de espacio y tiempo de manera que éstos pueden sufrir deformaciones de maneras que Lorentz no consideraba posibles. Con todo, las ecuaciones para cambiar entre marcos de referencia inerciales en el artículo de Einstein de 1905 y el de Lorentz de 1904 son las mismas. Como la deducción de consecuencias empíricas requieren el uso de las mismas ecuaciones, dichas consecuencias también son las mismas. Esto hace a este caso una instancia de subdeterminación de teorías científicas por la evidencia. Sin embargo, explicar por qué entonces en pocos años se formó un consenso, entre físicos alemanes, en favor de la relatividad especial ha sido tema de un amplio debate en historia y filosofía de la ciencia.

Una explicación del éxito de la relatividad especial señala que la hipótesis de contracción de Lorentz era ad hoc. Por ejemplo, Gerald Holton señalaba "[...] la hipótesis de contracción cuando fue hecha era clara y descaradamente ad hoc" (Holton 1969, p. 179; las cursivas son del original). Sin embargo, no parece que la hipótesis fuera $a d h o c$, ya que se apoyaba en otros supuestos sobre las interacciones moleculares. Además, los criterios para considerar ad hoc a una hipótesis también han resultado problemáticos, como muestra la investigación de Hunt (2012). Michel Janssen concluye que los intentos de mostrar que la hipótesis de contracción era ad hoc "han sido todos insatisfactorios" (Janssen 2002, p. 432).

Por su parte, Elie Zahar (1973a y 1973b) atribuye el éxito de la relatividad especial al éxito de la relatividad general. "Fue sólo cuando el programa de Einstein produjo la relatividad general que superó al de Lorentz empíricamente al explicar exitosamente la precesión anómala del perihelio de Mercurio" (Zahar 1973b, p. 259). Sin embargo, Feyerabend (1974) y Nugayev (1983) señalan, entre otras cosas, que Zahar sólo puede explicar el éxito de la relatividad especial hasta la publicación de la relatividad general en 1915, pero la relatividad especial quedó establecida antes, ${ }^{11}$ mejor de lo que estaría la relatividad general por muchos años. ${ }^{12}$

${ }^{11}$ De acuerdo con Stanley Goldberg: "1911 es significativo porque hay evidencia de que, al menos para los líderes en la ciencia, la teoría se pensaba como bien establecida para entonces" (Goldberg 1984, p. 182).

${ }^{12}$ El escepticismo en relación con la relatividad general no desapareció por completo aun después de las observaciones del eclipse de 1919 con las que se corroboró 
Literatura más reciente atribuye el éxito de la relatividad a que Lorentz fallara en hacer consistentes sus hipótesis con las explicaciones de Max Planck (1858-1947) acerca de la radiación del cuerpo negro:

A inicios del siglo XX, se reconoció que los estudios experimentales y teóricos de la radiación térmica estaban en conflicto con un modelo de éter como el asumido por Lorentz. De acuerdo con el así llamado "teorema de equipartición" de la teoría cinética del calor, tal éter, en equilibrio térmico con la materia, debería transmitir radiación electromagnética de todas las frecuencias posibles, atribuyéndosele a cada frecuencia una parte igual del total. De hecho, la velocidad de las partículas de luz no debería cambiar. Pero dado que no hay límite, en principio, para las frecuencias a las que el éter puede vibrar, contrario al caso de la materia ordinaria, el éter con sus infinitos grados de libertad eventualmente absorbería toda la energía de un sistema físico, de manera que un equilibrio térmico entre éter y materia se hace imposible. (Renn 2007, p. 45)

Como las explicaciones de Planck eran las únicas consistentes con los datos experimentales, el propio Lorentz reconoció, para 1908, la necesidad de revisar la electrodinámica en la que había trabajado. Mientras tanto, la relatividad especial era independiente de supuestos acerca de procesos como la radiación del cuerpo negro y hacía innecesario al éter. En pocas palabras, "Lo que eventualmente superó la teoría de Lorentz fue la teoría cuántica, no la teoría de la relatividad" (Janssen 2002, p. 431). Esta explicación parece convincente (aunque no exenta de dificultades), ${ }^{13}$ pero atribuye el éxito de la relatividad especial al camino que siguió la indagación temprana en la física

una de sus predicciones. Al parecer, Einstein recibió el premio Nobel por el efecto fotoeléctrico y no por la relatividad general debido a ello. Incluso para físicos de la generación de Thomas Kuhn, de mediados del siglo XX, la relatividad especial parecía mejor establecida que la general: "Yo estoy, sin embargo, bastante seguro de que para 1915, o muy poco después, la relatividad especial había sido aceptada profesionalmente y puesta en uso a un extremo que la relatividad general no había alcanzado treinta o cuarenta años después, cuando yo mismo era estudiante" (Kuhn 1980, p. 189).

${ }^{13}$ Se puede señalar, por ejemplo, que la electrodinámica clásica no dependía tanto del éter como se ha supuesto "Los méritos de su aproximación tienen [...] una base cuya apreciación no requiere compromiso con la fisicalidad del éter" (Brown 2005, p. 2). Es decir, "La dificultad se presenta porque las intuiciones dinámicas válidas de los pioneros como FitzGerald, Lorentz y Poincaré están contaminadas en la mente de la mayoría de los físicos por asociaciones con la filosofía del éter [... pero] el éter no desempeñó ningún papel realmente esencial en el pensamiento, previo a 1905, que llevó al descubrimiento de la contracción de longitudes y dilatación del tiempo [...] 
cuántica y no a ninguna virtud particular de las ideas de Einstein. La intuición de filósofos como Dewey o Brigman de que había algo profundamente innovador en el trabajo de Einstein sería poco más que una ilusión, o bien, esa novedad no desempeñaría ningún papel en el éxito de la relatividad. Esto llevó a Janssen (2002) a argumentar que, aunque acepta que la cuántica fue lo que superó a Lorentz, las ideas de Einstein eran mejores que las de Lorentz por razones similares a las que hicieron a las ideas de Copérnico preferibles a las de Ptolomeo.

[...] en ambos casos el argumento más fuerte en favor de la nueva interpretación, y en contra de la antigua, fue esencialmente un argumento de causa común, esto es, la nueva interpretación rastrea a una causa común lo que en la interpretación antigua eran coincidencias inexplicables. (Janssen 2002, p. 422)

Por ejemplo, Janssen señala que un cuerpo en movimiento sufre una contracción dada por un factor que depende de la velocidad de la luz y el tiempo sufre una dilatación dada por el mismo factor, lo cual es sólo una coincidencia para Lorentz, pero en la relatividad esto es consecuencia de cómo se utilizan segmentos de espacio-tiempo particulares para definir la longitud de un sistema o la duración de un proceso. ${ }^{14}$ Sin embargo, no parece claro en qué sentido el espacio-tiempo puede desempeñar el papel explicativo que Janssen le atribuye. "Pero apelar a la acción de una conexión de espacio-tiempo de fondo en el cual las partículas están inmersas - lo que Weyl llamaba el 'campo guía' - es, podría decirse, aumentar el misterio, no resolverlo" (Brown y Pooley 2004, p. 4).

Norton (2008) argumenta que, como Einstein obtiene las mismas consecuencias que Lorentz, en la teoría de este último hay elementos

Renunciar al prejuicio del éter como sustrato simplemente no implica rechazar la base dinámica de los argumentos de los pioneros o sus variaciones modernas" (Brown 2005, pp. 144-145). Además, es cuestionable que la relatividad especial realmente fuera independiente de supuestos sobre la radiación y el electrón, lo que se supone que es su ventaja respecto de Lorentz. Como señaló Wolfang Pauli: "La contracción de una barra de medición no es un proceso elemental, sino uno muy complicado. No tomaría lugar excepto para la covarianza con respecto al grupo de Lorentz de las ecuaciones básicas de la teoría del electrón, así como aquellas leyes, hasta ahora desconocidas para nosotros, que determinan la cohesión del mismo electrón" (Pauli 1958 [1921], p. 15).

${ }^{14}$ Una revisión más detallada de esta propuesta de Janssen y otras que se enfocan en factores no empíricos que pudieran haber favorecido a la relatividad es Acuña 2014. 
(o estructura) que son innecesarios y, por lo tanto, superfluos. Brown (2019) rechaza este argumento señalando que la estructura de la teoría de Lorentz, adicional a la que tiene Einstein, desempeña un papel esencial y no puede eliminarse sin cambios explicativos y de interpretación.

\section{Medición científica y el caso de Einstein contra Lorentz}

La elección entre la relatividad de Einstein y la electrodinámica de Lorentz está subdeterminado si consideramos que las consecuencias empíricas proporcionan la única manera de discernir entre ellas. Que se formara un rápido consenso en favor de Einstein se explica señalando conflictos entre el éter de la propuesta de Lorentz y la cuántica. Sin embargo, Brown (2005, pp. 144-145) señala que las conclusiones de Lorentz, Poincaré y otros no dependían tan fuertemente del éter. Además, la relatividad supone un cambio bastante radical y contraintuitivo en nuestra concepción del espacio y el tiempo. ¿Por qué sería preferible optar por esta nueva manera de entender al espacio y al tiempo que sólo modificar la propuesta de Lorentz?

Considero que se puede responder a esta pregunta tomando en cuenta el desarrollo de la medición científica. En las secciones 2 y 3 , señalé que a finales del siglo XIX había la posibilidad de utilizar la velocidad de la luz para resolver dificultades con las mediciones de grandes distancias y estandarizar el tiempo. También comenté que medir implica coordinar procesos con nuestro entendimiento de un concepto cuantitativo. Los filósofos del positivismo lógico consideraban que la coordinación se logra estipulando definiciones convencionales. Ya que no se pueden verificar empíricamente enunciados como "una regla para medir conserva su longitud al ser transportada", estos se toman como definiciones arbitrarias que regulan nuestro uso del concepto de longitud. Es decir, se establece por definición que la longitud de la regla en un sitio es la misma que en otro.

No es el concepto de igualdad de longitud lo que se ha de definir, sino que se ha de seleccionar un objeto real que corresponda a él. Una estructura física es coordinada con el concepto de igualdad de longitud, justamente como el metro estándar es coordinado con el concepto de unidad de longitud. (Reichenbach 1957, p. 16)

Sin embargo, otros estudios han señalado que es más adecuado reconocer que la coordinación en medición resulta de un desarrollo histórico en el que procesos de medición y conceptos cuantitativos se 
ajustan mutuamente. "La pregunta ¿qué constituye una medición de (una cantidad física) X? y ¿qué es (esa cantidad física) X? no pueden responderse independientemente una de otra" (van Fraassen 2008, p. 116). En una fase del desarrollo histórico la tecnología puede permitirnos mejorar el proceso por el cuál medimos valores asociados a conceptos cuantitativos para que cumpla mejor con los requerimientos de ese concepto; pero en otra fase lo que cambiamos es nuestra comprensión de los conceptos cuantitativos que medimos. Los conceptos e instrumentos de medición evolucionan de manera conjunta mediante un proceso escalonado en el que, en cada fase, uno se toma como estable para corregir al otro. Esto involucra tanto el reconocimiento de regularidades empíricas como decisiones guiadas por la búsqueda de coherencia. Estas regularidades empíricas no son fáciles de reconocer, ya que no tenemos intuición directa de, por ejemplo, la igualdad de dos instantes de tiempo o de longitudes espacialmente separadas. Podemos determinar si dos varas tienen la misma longitud en un momento dado si las colocamos una junto a la otra; también podemos determinar que dos relojes están sincronizados observándolos juntos; pero no podemos determinar de manera directa si dos varas en tiempos o lugares diferentes tienen la misma longitud, o si dos relojes siguen sincronizados cuando se alejan uno del otro. Uno puede escoger el proceso de medición que defina unidades de tiempo iguales, pero esa decisión está limitada por requerimientos de coherencia, tales como que dos relojes sincronizados, que coinciden espacialmente y que avanzan al mismo ritmo, deben mantenerse sincronizados. Determinar si lo que debemos modificar es el proceso de medición, o nuestra concepción de aquello que queremos medir depende de la situación histórica específica.

Lo que faltaba en las prácticas de medición a inicios del siglo XX era un cambio conceptual y es por ello que Einstein comienza su artículo de 1905 con una reflexión sobre el concepto de simultaneidad. Este análisis es pertinente porque la física de la época reconoce la estabilidad de la medición de la velocidad de la luz, pero no la del espacio y el tiempo. En contraste, Lorentz no hace esa revisión conceptual, sino que continúa usando conceptos cuyo uso no estaba apropiadamente regulado por procesos confiables de medición. La robustez en la estabilidad de la velocidad de la luz no presta el mismo apoyo a Lorentz que a Einstein porque ésta desempeña papeles diferentes en ambos casos. Lorentz no la usa para regular su uso de los conceptos, de hecho, podría haber prescindido de ella, pero sí depende de conceptos que requieren ajustes que él no provee. Aunque Lorentz no negaba la estabilidad de la velocidad de la luz, 
consideraba que sólo las interacciones electromagnéticas dan un valor estable para esta velocidad, ya que no permiten detectar el movimiento de observadores respecto al éter. "Para otros fenómenos, no hay duda de que Lorentz aun creía que el movimiento con respecto al éter podía, en principio, ser detectado" (Darrigol 2005, p. 12). También Poincaré, aunque señaló que el tiempo local sería el que mediría un observador en movimiento respecto a otro al sincronizar sus relojes con señales de luz y reconocía que "Las propiedades del tiempo son, por tanto, meramente las de nuestros relojes, así como las propiedades del espacio son meramente las de nuestros instrumentos de medición" (Poincaré 1963 [1913], p. 18); nunca llegó a adoptar por completo las consecuencias de estas ideas.

En otras palabras, las prácticas de medición generaron la necesidad de un cambio conceptual en la física de inicios del siglo XX; lo que Einstein hace, pero no Lorentz. La innovación de Einstein consiste, no en hacer sinónimos a los conceptos con sus mediciones, como sugería Bridgman, sino en incorporar la robustez que había alcanzado la medición de la velocidad de la luz para regular el uso de otros conceptos.

La forma de proceder de Einstein es superior a la de Lorentz porque tiene un fundamento más robusto, lo que permite que la relatividad especial sobreviva a los profundos cambios por los que pasó la física de inicios del siglo XX. Quine (1976) señalaba que siempre hay diferentes maneras en las que podemos modificar nuestras creencias para preservar una teoría o hipótesis. ${ }^{15}$ En principio, esto podría haberse hecho para rescatar la teoría de Lorentz. Sin embargo, el mismo Quine reconocía que, en la práctica, hay aspectos de la ciencia que estamos menos dispuestos a modificar. ${ }^{16}$ Ya sea que los eventos entre 1905 y 1911 fueran suficientes para refutar por completo a Lorentz o no, la estabilidad de la medición de la velocidad de la luz era más confiable que la concepción clásica del espacio y el tiempo. Por la misma razón, los cambios teóricos y conceptuales que los físicos tuvieron que considerar para dar cuenta de los fenómenos que abordó la física cuántica tampoco involucraron una revisión sustancial de la relatividad especial. Hubo más disposición a modificar la electrodinámica que la relatividad. No es, necesariamente, que la relatividad especial fuera independiente de supuestos sobre la radiación y las interacciones moleculares, más bien, es el uso de conceptos

${ }^{15}$ Véase en especial la sección 6 de ese artículo de Quine.

${ }^{16}$ Quine pensaba que, aunque posible, era poco viable modificar las matemáticas, la lógica o enunciados cercanos a la experiencia sensible para preservar una teoría, considero que lo mismo ocurre con prácticas estables de medición científica. 
definidos a partir de operaciones confiables de medición lo que la hace lo suficientemente robusta como para sobrevivir la emergencia de la física cuántica.

Explicar el éxito de la relatividad especial como parte de los cambios en las prácticas de medición tiene la ventaja de recuperar las intuiciones de filósofos como Bridgman y Dewey. Como señala el primero, redefinir conceptos a partir de operaciones de medición es lo que hace a la relatividad de Einstein superior, pero no necesariamente porque el operacionalismo, como se conoce a la postura filosófica de Bridgman, sea una adecuada teoría del significado de conceptos científicos, ${ }^{17}$ sino por la estabilidad que pueden alcanzar las prácticas de medición científica. Por otra parte, lo presentado aquí es consistente con Dewey, quien consideraba que el trabajo de Einstein "[...] marcó un final, al menos en lo que concierne a la ciencia natural, en los intentos de enmarcar concepciones científicas en términos de propiedades asignadas a aquellos objetos independientemente de las consecuencias observadas de una operación experimental" (Dewey 1929, pp. 145-146).

\section{Conclusión}

El objetivo de este artículo fue indagar si el estudio filosófico de la medición científica permite resaltar algún aspecto distintivo de la relatividad especial que explique su éxito en contraste con la electrodinámica de Lorentz. El aspecto distintivo de la relatividad especial que explica, al menos, parte de este éxito se encuentra en la relación entre conceptos científicos y las prácticas de medición de los mismos. Si reconocemos que la coordinación entre ambos es producto de un desarrollo histórico que genera la necesidad de cambiar tanto conceptos como procesos, y no de una estipulación única en un momento dado, podemos concluir que a inicios del siglo XX había la necesidad de un cambio conceptual. El hecho de que Einstein responda a esa necesidad hace a la relatividad preferible sobre la teoría de Lorentz, ya que le da una mayor capacidad de síntesis. Esta capacidad se hace evidente al considerar cómo afectaba las prácticas de medición; permitía establecer un valor para la órbita de la Tierra coherente con las mediciones terrestres de la velocidad de la luz y proporcionaba una opción natural para la estandarización del tiempo. Además, la relatividad mostraba que el carácter absoluto o relativo

${ }^{17}$ Chang (2004 y 2009) analiza algunas de las objeciones pertinentes al operacionalismo como teoría del significado, pero propone también maneras diferentes de entenderlo. 
del espacio y del tiempo requiere justificación. Einstein ofrecía esa justificación a partir de operaciones de medición caracterizadas por una robusta confiabilidad y estabilidad. Este tipo de ventajas son consideraciones que hacen viable superar casos de subdeterminación en ciencia y, en este caso, explican que los profundos cambios conceptuales que generó la emergencia de la física cuántica no afectaran a la relatividad especial y sí a la electrodinámica de Lorentz cuyo fundamento era menos estable.

\section{BIBLIOGRAFÍA}

Acuña, P., 2014, "On the Empirical Equivalence between Special Relativity and Lorentz's Ether Theory", Studies in History and Philosophy of Science Part B - Studies in History and Philosophy of Modern Physics, vol. 46, no. 1, pp. 283-302.

Acuña, P. y D. Dieks, 2014, “Another Look at Empirical Equivalence and Underdetermination of Theory Choice", European Journal for Philosophy of Science, vol. 4, no. 2, pp. 153-180.

Berka, K., 1983, Measurement. Its Concepts, Theories and Problems, Reidel Publishing Company, Dordrecht, Holland.

Boyer, C.B., 1941, "Early Estimates of the Velocity of Light", Isis, vol. 33, no. 1, pp. 24-40.

Bradley, C., 2021, "The Non-Equivalence of Einstein and Lorentz", The British Journal for the Philosophy of Science, vol. 72, no. 4, https://doi.org/10.1093/bjps/axz014 [fecha de consulta: 10/05/2020].

Bridgman, P.W., 1970, "Einstein's Theories and the Operational Point of View", en P.A. Schilpp (comp.), Albert Einstein Philosopher-Scientist, Open Court, Nueva York, pp. 335-354.

Brown, H.R. y O. Pooley, 2004, "Minkowski Space-Time: A Glorious NonEntity", http://philsci-archive.pitt.edu/id/eprint/1661 [fecha de consulta: $10 / 05 / 2020]$.

Brown, H.R., 2005, Physical Relativity. Space-Time Structure from a Dynamical Perspective, Oxford University Press, Nueva York.

Brush, S., 1999, "Why Was Relativity Accepted?", Physics in Perspective, vol. 1, no. 2, pp. 184-214.

Camp, W.V., 2011, "On Kinematic versus Dynamic Approaches to Special Relativity", Philosophy of Science, vol. 78, no. 5, pp. 1097-1107.

Canales, J., 2015, The Physicist and the Philosopher. Einstein, Bergson, and the Debate that Changed our Understanding of Time, Princeton University Press, Nueva Jersey.

Canales, J., 2009, A Tenth of a Second: A History, The University of Chicago Press, Chicago y Londres. 
Cassidy, D., 1986, "Review: Understanding the History of Special Relativity", Historical Studies in the Physical and Biological Sciences, vol. 16, no. 1, pp. 177-188.

Chang, H., 2012, Is Water H2O? Evidence, Realism and Pluralism, Springer, Nueva York.

Chang, H., 2009, Operationalism, https://plato.stanford.edu/archives/fall2009/entries/operationalism/ [fecha de consulta: 07/02/2020].

Chang, H., 2004, Inventing Temperature: Measurement and Scientific Progress, Oxford University Press, Nueva York.

Darrigol, O., 2005, "The Genesis of the Theory of Relativity", en T. Damour, O. Darrigol, B. Duplantier, V. Rivasseau (comps.), Einstein, 1905-2005 Poincaré Seminar 2005, Birkhäuser Basel, Boston, pp. 1-31.

Darrigol, O., 2000, Electrodynamics from Ampere to Einstein, Oxford University Press, Nueva York.

Dewey, J., 1929, The Quest for Certainty: A Study of the Relation of Knowledge and Action, Minton, Balch and Company, Nueva York.

Dorling, J., 1966, "Length Contraction and Clock Synchronisation: The Empirical Equivalence of the Einsteinian and Lorentzian Theories", en British Journal for the Philosophy of Science, vol. 19, no. 1, pp. 67-69.

Einstein, A., 2012 [1923], "How I Created the Theory of Relativity?", en D.K. Buchwald, J. Illy, Z. Rosenkranz y T. Sauer (comps.), The Collected Papers of Albert Einstein. vol. 13, Doc. 399, Princeton University Press, Princeton, Nueva Jersey, pp. 636-641.

Einstein, A., 2005 [1905], "Sobre la electrodinámica de cuerpos en movimiento", trad. Javier García Sanz, en J.M. Sánchez Ron (comp.), Albert Einstein, Crítica, Barcelona, pp. 399-430.

Feyerabend, P.K., 1974, "Zahar on Einstein", The British Journal for the Philosophy of Science, vol. 25, no. 1, pp. 25-28.

Feigl, H., 1974, "Empiricism at Bay?", en R.S. Cohen y M.W. Wartofsky (comps.), Methodological and Historical Essays in the Natural and Social Sciences, D. Reidel Publishing Company, Dordrecht, Holland, pp. 1-20.

Fizeau, H., 1851, "Sur les hypothèses relatives à l'éther lumineux, et sur une expérience qui paraît démontrer quele mouvement des corps change la vitesse avec laquelle la lumière se propage dans leur intérieur", Comptes Rendus de l'Académie des sciences, no. 33, pp. 349-355.

Fizeau, H., 1849, "Sur une expérience relative à la vitesse de propagation de la lumière", Comptes Rendus de l'Académie des sciences, no. 29, pp. 90-92.

Galison, P., 2005, Relojes de Einstein, mapas de Poincaré, Crítica, Barcelona.

Galison, P., 1997, Image and Logic. A Material Culture of Microphysics, The University of Chicago Press, Chicago. 
Goldberg, S., 1984, Understanding Relativity. Origin and Impact of a Scientific Revolution, Birkhauser, Boston, Basel, Stuttgart.

Grünbaum, A., 1959, "The Falsifiability of the Lorentz-Fitzgerald Contraction Hypothesis", British Journal for the Philosophy of Science, vol. 10, no. 37 , pp. 48-50.

Guillaumin, G.E., 2016, Génesis de la medición celeste. Una historia cognitiva del crecimiento de la medición en astronomía, Tirant Humanidades, Ciudad de México.

Hertz, H., 1896, “On the Relations between Light and Electricity, 1889”, en P. Lenard (comp.), Miscellaneous Papers by Heinrich Hertz, Macmillan and Co., Nueva York, pp. 313-327.

Holton, G., 1969, "Einstein, Michelson, and the 'Crucial' Experiment", Isis, vol. 60 , no. 2 , pp. 132-197.

Hunt, C., 2012, "On Ad Hoc Hypotheses", Philosophy of Science, vol. 79, no. 1 , pp. 1-14.

Janssen, M., 2019, "How Did Lorentz Find His Theorem of Corresponding States?", Studies in History and Philosophy of Science Part B: Studies in History and Philosophy of Modern Physics, vol. 67, pp. 167-175.

Janssen, M., 2002, "Reconsidering a Scientific Revolution: The Case of Einstein versus Lorentz", Physics in Perspective, vol. 4, no. 4, pp. 421446.

Janssen, M., 1995, A Comparison between Lorentz's Ether Theory and Special Relativity in the Light of the Experiments of Trouton and Noble, tesis doctoral, University of Pittsburgh, Pittsburgh, E.U.A.

Kuhn, T.S., 1982, "La función de la medición en la física moderna”, en T.S. Kuhn (comp.), La tensión esencial. Estudios selectos sobre la tradición y el cambio en el ámbito de la ciencia, Fondo de Cultura Económica, México, pp. 202-247.

Kuhn, T.S., 1980, "The Halt and the Blind: Philosophy and History of Science", The British Journal for the Philosophy of Science, vol. 31, no. 2, pp. 181-192.

Le Verrier, U., 1872, "Sur les masses des planétes et la parallaxa du Soleil", Comptes rendus hebdomadaires des séances de l'Académie des sciences Mémoires et Comunications, T. LXXV, pp. 165-172, https://gallica.bnf.fr/ark:/12148/bpt6k30321/f167.item [fecha de consulta: $21 / 01 / 2020]$.

Lorentz, H.A., 1952 [1904], "Electromagnetic Phenomena in a System Moving with any Velocity Less Than That of Light", en A. Sommerfeld (comp.), The Principle of Relativity, Dover Publications INC., pp. 9-34.

Lorentz, H.A., 1895, "Attempt of a Theory of Electrical and Optical Phenomena in Moving Bodies", https://archive.org/details/Lorentz [fecha de consulta: 29/02/2016].

Lorentz, H.A., 1892, "La théorie électromagnétique de Maxwell et son application aux corps mouvants", Archives Néerlandaises des Sciences Exactes et Naturelles, T. XXV, pp. 1-190. 
Lorentz, H.A., 1886, “De l'influence du mouvement de la terre sur les phénomènes lumineux", Archives Néerlandaises des Sciences Exactes et Naturelles, T. XXI, pp. 7-176.

Mari, L., 2003, "Epistemology of Measurement", Measurement, vol. 34, no. 1, pp. 17-30.

Maxwell, J.C., 1881, A Treatise on Electricity and Magnetism, Vol. II, Oxford University Press, Londres.

McCormmach, R., 1980a, "Hertz", en C. Gillispie (comp.), Dictionary of Scientific Biography, Vol. 6, Simon and Schuster Macmillan, Nueva York, pp. 340-350.

McCormmach, R., 1980b, "Lorentz", en C. Gillispie (comp.), Dictionary of Scientific Biography, Vol. 8, Simon and Schuster Macmillan, Nueva York, pp. 487-500.

Michelson, A.A., 1881, "The Relative Motion of the Earth and the Luminiferous Ether", American Journal of Science, vol. 22, pp. 120-129.

Michelson, A.A. y E.W. Morley, 1887, "On the Relative Motion of the Earth and the Luminiferous Ether", American Journal of Science, vol. 34, pp. 333-345.

Michelson, A.A. y E.W. Morley, 1886, "Influence of the Motion of the Medium on the Velocity of Light", American Journal of Science, vol. 31, pp. 377-386.

Miller, A.I., 1981, Albert Einstein's Special Theory of Relativity. Emergence (1905) and Early Interpretation (1905-1911), Addison-Wesley, Reading Massachusetts.

Norton, J.D., 2008, "Must Evidence Underdetermine Theory?", en D. Howard, M. Carrier y J. Kourany (comps.), The Challenge of the Social and the Pressure of Practice. Science and Values Revisted, University of Pittsburgh Press, Pittsburgh, pp. 17-44.

Nugayev, R.M., 1983, "The History of Quantum Theory as a Decisive Argument Favoring Einstein over Lorentz", Philosophy of Science, vol. 52, no. 1, pp. 44-63.

Pauli, W., 1958 [1921], Theory of Relativity, Pergamon Press, Bristol.

Peirce, C.S., 1878, "How to Make our Ideas Clear", Popular Science Monthly, vol. 12, pp. 286-302.

Poincaré, H., 1913 [1898], "The Measure of Time", en H. Poincaré (comp.), The Foundations of Science, The Science Press, Nueva York, pp. 222234.

Poincaré, H., 1963 [1913], "Space and Time”, en H. Poincaré (comp.), Mathematics and Science: Last Essays (Dernières Pensés), Dover, Nueva York, pp. 15-24.

Poincaré, H., 2007 [1906], "On the Dynamics of the Electron (Excerpts)", en J. Renn y M. Schemmel (comps.), The Genesis of General Relativity, vol. 3, Gravitation in the Twilight of Classical Physics, Springer, Dordrecht, Holland, pp. 21-80, pp. 253-271. 
Pyenson, L., 1985, The Young Einstein. The Advent of Relativity, Adam Hilder, Bristol y Boston.

Quine, W.V.O., 1976, "Two Dogmas of Empiricism", en S. Harding (comp.), Can Theories be Refuted? Essays on the Duhem-Quine Thesis, D. Reidel Publishing Company, Boston, pp. 41-64.

Reichenbach, H., 1957, The Philosophy of Space and Time, Dover, Nueva York.

Renn, J., 2007, "Classical Physics in Disarray. The Emergence of the Riddle of Gravitation", en M. Janssen, J.D. Norton, J. Renn, T. Sauer, J. Stachel y L. Divarci (comps.), The Genesis of General Relativity, vol. 1: Einstein's Zurich Notebook: Introduction and Source, Springer, Dordrecht, Holland, pp. 21-80.

Staley, R., 2002, "Travelling Light", en Bourguet, Licoppe y Sibum (comps.), Instruments, Travel and Science. Itineraries of Precision from the Seventeenth to the Twentieth Century, Routledge, Londres, pp. 243272.

Tal, E., 2012, The Epistemology of Measurement: A Model-Based Account, Ph.D. Dissertation, Graduate Department of Philosophy, University of Toronto, http://hdl.handle.net/1807/34936 [fecha de consulta: 04/07/2016].

Van Fraassen, B.C., 2008, Scientific Representation: Paradoxes of Perspective, Oxford University Press, Nueva York.

Zahar, E., 1973a, "Why Did Einstein's Programme Supersede Lorentz's? (I)", The British Journal for the Philosophy of Science, vol. 24, no. 2, pp. 95-123.

Zahar, E., 1973b, “Why Did Einstein's Programme Supersede Lorentz's? (II)", The British Journal for the Philosophy of Science, vol. 24, no. 2, pp. 223-262.

Recibido el 8 de febrero de 2020; aceptado el 21 de mayo de 2021. 